\title{
Perception by monkeys II. Use of cues at a distance by young and old monkeys'
}

\author{
Koger T. Davis, Arthur Lampert and David E. Rumelhart ${ }^{2}$ \\ UNIVERSITY OF SOUTH DAKOTA
}

\begin{abstract}
Abstraet
Fourteen rhesus monkeys and two human Os were trained to discriminate between identical blocks of wood placed 13 in apart, using cues that were provided by a pointer that was placed at random in positions spaced 1.0 in apart between the manipulanda. Monkeys made increasingly more errors as a function of increasing distance between the manipulandum and discriminandum, and extensive practice did not alter this relationship. The human Os, however, made no errors at positions of the pointer other than the center.
\end{abstract}

\section{Problem}

There is a considerable body of literature which indicates that monkeys do not respond well to cues that are remote from the reward (McClearn \& Harlow, 1954; Murphy \& Miller, 1955; 1956; 1958; Shuck et al., 1961; McDowell, 1958; Meyer et al., 1961), and rewards decrease in value sharply as a function of distance (Davis, 1958). Furthermore, Miller and Murphy (1964) showed that separation of response from reward and cue interferes with performance more than separation of reward from cue and response. The following experiment employed monkeys with humans as controls to determine the degree of continuity or discontinuity of response as a function of the remoteness of cue and manipulandum. It was also possible to determine whether or not discontinuity depended on practice. If there was continuity of performance, the perception would be a special case of generalization; if there was discontinuity after practice, the perception would be analogous to learning set; but invariate discontinuity would be evidence that the subject was employing some form of mediation to bridge the spatial gap.

\section{Method}

Fourteen highly trained rhesus monkeys and two undergraduate college students were used as Ss. Eight of the monkeys were aged approximately 12.0-13.0 yr. at the beginning of the experiment. Four of these had received $1100 \mathrm{r}$ whole-body radiation during a period 6.5-9.5 yr. before the present experiment and five had been nonirradiated controls. The remaining five were progeny of the older Ss, were aged 4.5-6.5, and had followed the same program of experimentation as the old monkeys during the three years that preceded this study.

The unique apparatus for the problem fitted into the stimulus tray carrier of the WGTA and consisted of a black board presented to Ss at a $50^{\circ}$ angle. It contained two foodwells, 14 in apart side-to-side, each covered with 2-in white squares of Masonite that were positioned exactly on every trial. A pointer made of 9 gauge wire, also painted white, was attached to a calibrated vernier in the rear of the apparatus and extended parallel to the sides of the squares. It could be moved by $\mathrm{E}$ until it was adjacent to one or the other square, or it could be placed at any intermediate point. Arbitrarily, 13 pointer readings were selected. Number 13 represented placement next to one of the stimuli, No. 1 next to the other and 7 was intermediate. The other readings were at 1.0 in intervals between the stimuli.

Each $\mathrm{S}$ was given preliminary training until it met a criterion of 45 trials correct out of 50 a day. On any trial the wire pointer was parallel to and touching the square on the left, $\mathrm{S}$ was rewarded in the lefthand foodwell. If the pointer was on the opposite side of the board, touching the square on the right, the righthand stimulus was reinforced.

Critical training consisted of three randomizations a day of the 13 possible positions. The first time Ss received critical training they reverted to position habits and were given four days training, 50 trials a day in which the pointer was placed either 3.0 in from the left or righthand square and reinforced. Then all Ss were returned to preliminary training, and all Ss met the criterion for the second time. The Ss resumed critical training in which, as before, the pointer was placed at any of the variable positions and response to either object was rewarded.

The experiment was replicated eight months after it was completed the first time, with the following changes. Each S received 200 trials of training in which the pointer was placed at positions 3.0 in from either the left or righthand stimulus and correct responses were reinforced. Critical training consisted of 50 trials at each pointer position, with the positions of the pointer randomized.

\section{Results and Diseussion}

Figure 1 shows the performance of subhuman and human Os on discriminations based on remote cues provided without differential reinforcement. The two positions nearest the manipulanda on either side are designated Near, the two on the left and the two on the righthand side of center designated Far, and the intermediate two positions on each side, Intermediate. Performance of monkeys clearly depended on generalization; the closer the pointer was to one of the manipulanda the more correct responses. However, human Os, even though they were trained in the same manner as monkeys, experienced no more difficulty using the cue when it was in the far than the near positions, the degree 


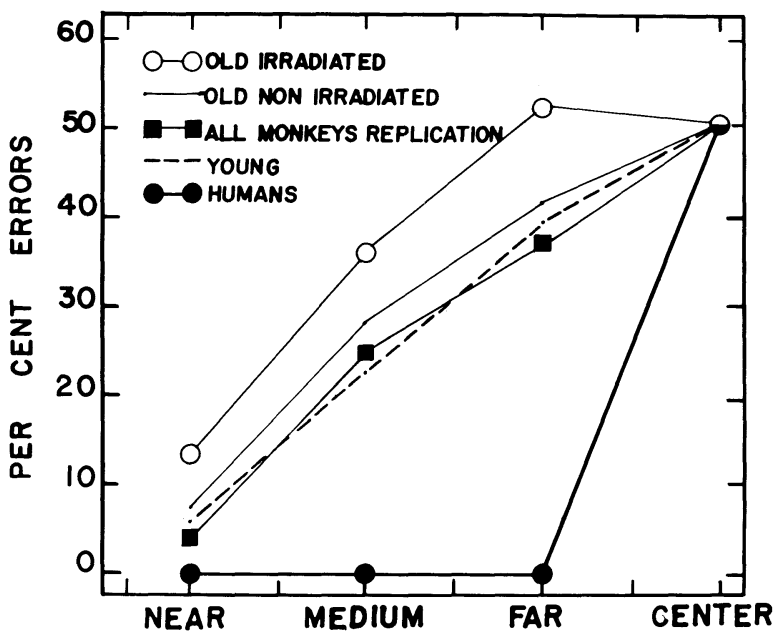

Fig. 1 Performance as a function of distance between manipulanda and discriminanda.

of generalization being equivalence, and mediation undoubtedly verbal.

The three groups of monkeys performed in the direction that would be predicted by the hypothesis that the deleterious effects of irradiation are additive to those of natural aging. The difference between the performance of the old survivors of radiation and their young and old nonirradiated controls are significantly different at the medium and far positions $(U=5$ and 0 respectively, $\mathrm{n}_{1}=4, \mathrm{n}_{2}=14, \mathrm{p}<.05$ and .002$)$ and closely approaches significance in the near positions $(U=6)$. The old nonirradiated monkeys and the young animals, however, do not differ significantly at any of the positions or in overall number of responses. Since earlier work with these Ss indicated that those performing best in several common laboratory tests were more likely to die of radiation death than poor performers (Davis \& Steele, 1963) the difference between these survivors and the other Ss may be related to the mechanism that was selected in death. Considering the nature of the present task it would seem appropriate to investigate further the likelihood that the mechanism involves attention. This is supported by the interpretations that McDowell and Brown (1963) have made of differences in the performance of irradiated male and female monkeys on problems with reduced peripheral cues.

When Ss received additional training preceding the replication and were reinforced at 3-in intervals between the pointer and correct stimulus, the young animals benefited by the experience whereas the old Ss did not, the difference being highly significant during the last block of 50 trials $\left(\mathrm{U}=0, \mathrm{n}_{1}=5, \mathrm{n}_{2}=9, \mathrm{p}<.002\right)$. However, performance of young and old Ss did not differ significantly during the replication of critical training on any of the pointer positions.

\section{References}

DAVIS, R. T. Linear position preference habits of monkeys. J. genet. Psychol., 1958, 91, 233-238.

DAVIS, R. T., \& STEELE, J. P. Performance selection through radiation death in rhesus monkeys. J. Psychol., 1963, 56, 119-136.

McCLEARN, G. E., \& HARLOW, H. F. The effect of spatial contiguity on discrimination learning by rhesus monkeys. J. comp. physiol. Psychol., 1954, 47, 391-394.

McDOWELL, A. A. Transfer by normal and chronic whole-body irradiated monkeys of a single learned discrimination along a peripheral cue gradient. J. genet. Psychol., 1960, 97, 41-58.

McDOWELL, A. A., \& BROWN, W. L. Sex and radiation as factors in peripheral cue discrimination learning. J. genet. Psychol., 1963, 102, 261-265.

MEYER, D. R., POLIDORA, V. J., \& McCONNELL, D. G. Effects of spatial S-R contiguity and response delay upon discriminative performance by monkeys. J. comp. physiol. Psychol., 1961, 54, 175-177.

MILLER, R. E., \& MURPHY, J. V. Discrimination learning with vertical vs. horizontal stimulus relationships. J. comp. physiol. Psychol., 1956, 49, 80-83.

MILLER, R. E., \& MURPHY, J. V. Influence of the spatial relationships between the cue, reward, and response in discrimination learning. J. exp. Psychol., 1964, 67, 120-123.

MURPHY, J. V., \& MILLER, R. E. The effect of spatial contiguity of cue and reward in the object-quality learning of rhesus monkeys. J. comp. physiol. Psychol., 1955, 48, 211-229.

MURPHY, J. V., \& MILLER, R. E. Spatial contiguity of cue, reward, and response in discrimination learning by children. J. exp. Psychol., 1959, 58, 485-489.

SCHUCK, J. E., POLIDORA, V. J., McCONNELL, D. G., \& MEYER, D. R. Response location as a factor in primate discrimination learning. J.comp. physiol. Psychol., 1961, 54, 543-545.

\section{Notes}

1. This investigation was supported in part by Public Health Service Research Grant MH-07147-01, from the Institute of Mental Health and Grant RH-66, C3 from the Division of Radiological Health, Bureau of State Services, Public Health Service.

2. The third author is currently a graduate student at Stanford University. 